\title{
Propuesta estratégica de cooperación para impulsar la calidad académica en la Facultad de Ciencias Económicas y Administrativas de la Universidad Nacional Toribio Rodríguez de Mendoza de Amazonas, 2017
}

\section{Strategic proposal for cooperation to promote academic quality at the Facultad de Ciencias Económicas y Administrativas of the Universidad Nacional Toribio Rodríguez de Mendoza de Amazonas, 2017}

\author{
Jonathan Antonio Huaman Malqui ${ }^{1}$, Herber Sedillo Llanca ${ }^{2}$
}

\begin{abstract}
RESUMEN
Este trabajo de investigación se realizó con el objetivo de elaborar una propuesta estratégica de cooperación para la Facultad de Ciencias Económicas y Administrativas de la Universidad Nacional Toribio Rodríguez de Mendoza de Amazonas (UNTRM); para esta investigación se ha tomado una muestra de 35 estudiantes del X ciclo entre las escuelas de Administración de Empresas y Turismo y Hostelería. Se planteó el problema, ¿Es factible la propuesta de cooperación para fortalecer la calidad académica de la Facultad de Ciencias Económicas y Administrativas de la UNTRM? para responder se ha empleado instrumentos de recolección de datos por encuesta y el método descriptivo, la hipótesis planteada es la elaboración de la propuesta estratégica de cooperación, y servirá como guía para que la facultad pueda establecer cooperación, mejorar la calidad académica y lograr el fortalecimiento de la universidad en conjunto. Los resultados son evidentes, debido a la necesidad de internacionalización de las escuelas; más del 50\% de los estudiantes ven la necesidad urgente de salir a pasantías y el $40 \%$ busca permanecer en la región Amazonas. Por tal motivo, esta investigación concluye en la importancia y beneficios futuros de optar por la cooperación, aceptando la hipótesis de la presente investigación.
\end{abstract}

Palabras clave: Cooperación, fortalecimiento, calidad, educación, formación.

\begin{abstract}
This research work was carried out with the objective of preparing a strategic cooperation proposal for the Faculty of Economic and Administrative Sciences of the Toribio Rodríguez of Mendoza National University of Amazonas (UNTRM); for this research, a sample of 35 students of the X cycle between the schools of Business Administration and Tourism and Hospitality was taken. The problem was raised, is the cooperation proposal to strengthen the academic quality of the Faculty of Economic and Administrative Sciences of the UNTRM feasible? In order to respond to the data collection instruments by survey and the descriptive method, the proposed hypothesis is the formulation of the strategic cooperation proposal, which serves as a guide for the faculty to establish cooperation, improve academic quality and achieve the strengthening of the university as a whole. The results are evident, due to the need for internationalization of schools; more than $50 \%$ of the students to the urgent need to go out for internships and $40 \%$ seek to stay in the Amazon region. For this reason, this research concludes on the importance and future benefits of opting for cooperation, accepting the hypothesis of the present investigation.
\end{abstract}

Keywords: Cooperation, strengthening, quality, education, training. 


\section{INTRODUCCIÓN}

Este trabajo de investigación, está basado en antecedentes que describen los objetivos en común, la elaboración de la propuesta estratégica se sustenta con el diagnóstico situación a través del FODA que se ha elaborado tomando en consideración los datos recolectados empleados con nuestros instrumentos además de la experiencia empírica que hemos adquirido al pasar los años como estudiantes, este estudio se realiza con la finalidad de contar con un documento guía para la facultad que sea capaz de orientar en gestiones de cooperación educativa universitaria para fortalecer las escuelas profesionales.

La Universidad Nacional Toribio Rodríguez de Mendoza es una universidad en crecimiento y prestigio nacional e internacional; Amazonas por décadas ha sido dirigida por personajes ajenos a la realidad regional sin compromiso y sin visiones, donde el progreso se veía extinto; en el año 2000, con la universidad se posicionó nuevos aires en la región y hoy existen ya profesionales dirigiendo los destinos de la región, es lógico que los recursos económicos son limitados, específicamente por ese problema se plantea la cooperación como medida para alcanzar mejor la calidad y mayores visiones de desarrollo profesional. En este estudio se precisa específicamente la cooperación de educación universitaria haciendo uso de la cooperación técnica e internacional en rubros de fortalecimiento universitario en la facultad de Ciencias Económicas y Administrativas de la UNTRM.

De lo descrito en el párrafo anterior, precisamos que esta investigación será una guía para alcanzar calidad académica, y que al mismo tiempo beneficiará a los docentes y estudiantes en el ámbito de la preparación profesional como tal.

La Agencia Peruana de Cooperación Internacional (APCI) menciona que para alcanzar niveles académicos se ha priorizado tratados y convenios tripartitos es decir la Universidad beneficiaria, el estado peruano y la fuente cooperante de manera que en conjunto asuman responsabilidades para alcanzar objetivos; no obstante, se puede hacer convenios interinstitucionales lo que responsabiliza a ambas instituciones tal como se especifiquen en los documentos que se suscriban. Para (Morles, 1997) se podría definir a la cooperación académica como un proceso humano intencional mediante el cual se actúa o trabaja con otro u otros, con el fin de lograr un beneficio mutuo en materia científica a partir de compartir conocimientos, experiencia, equipos, información y personal calificado. Las actividades de cooperación académica asumen una importancia vital, por cuanto ellas permiten vincularlos a otras realidades, economizar recursos, enfrentar tareas más complejas y, con ello, aumentar la pertinencia de las acciones que se realizan.

\section{MATERIAL Y MÉTODOS}

El presente estudio de investigación es un diseño descriptivo simple de tipo transaccional de una sola casilla, ya que se buscó recolectar, analizar y comparar la información de la variable en un momento dado o tiempo único, con el fin de poder describir la variable de estudio e indicadores de estudio.

La población en estudio estuvo constituido por los estudiantes del décimo ciclo de la Escuela Profesional de Administración de Empresas y Turismo y Hostelería (Administración en Turismo); los estudiantes de Economía y Contabilidad no se cuentan debido a que aún al 2017 - II no hay alumnos en el X ciclo. Por lo cual tenemos a 35 alumnos según información brindada por la Dirección General de Admisión y Registros Académicos

La muestra en estudio fue igual a la población, ya que dicha población es pequeña para este estudio y al estimar la muestra para el estudio se aproximaría a la población: se aplicó un muestreo no probabilístico (por conveniencia), ya que se tomará y evaluará al total poblacional a los estudiantes del décimo ciclo de las escuelas profesionales de Administración de Empresas y Turismo y Hostelería (Administración en Turismo).

Para realizar la investigación se empleó la estadística para el entendimiento del análisis de datos y para seleccionar las conclusiones generales más adecuadas a partir de datos parciales y representativos.

Para determinar la percepción de los estudiantes y docentes con respecto a la cooperación se empleó algunas técnicas e instrumentos, tales como, la encuesta y entrevista las cuales se basaron en los atributos o estándares siguientes.

- Oportunidad

- Seguridad

- Eficacia

-Accesibilidad

- Integralidad

- Trabajo en equipo

- Participación social

- Respeto a estudiantes 
- Información completa

- Satisfacción del usuario externo

La estructura que se fue utilizado para el cuestionario consta de la siguiente forma:

- Datos generales.

- Cooperación Académica

- Cooperación para el desarrollo

- Alternativas de cooperación para la facultad

Además; se diseñó una ficha de entrevista que se aplicó al decano de la Facultad de Ciencias Económicas y Administrativas.

Los procedimientos que se tomaron en cuenta en la investigación son los siguientes:

1. Se diseñó y elaboró los instrumentos de recolección de datos (cuestionario).

2. Se validó el instrumento de recolección de datos con una muestra piloto. La validación del instrumento se realizará teniendo en cuenta la evidencia relacionada con el contenido.

La validez de contenido se refiere al grado en que un instrumento refleja un dominio específico de contenido de lo que se mide (Hernández, Fernández \& Baptista, 2012, p. 247).

3 Se estimó el tamaño de muestra según la población, el tipo de muestreo del estudio.

4. Se administró y aplicó el cuestionario según la muestra en estudio.

5. Se procesó y tabuló la información obtenida con ayuda de la hoja de cálculo Microsoft Excel 2016.

6. Se organizó y presentó la información de los resultados mediante cuadros y gráficos estadísticos, según los objetivos de estudio.

7. Luego, se analizó, discutió y comparó los resultados obtenidos.

8. Se concluyó según los objetivos de estudio de investigación.

9. Finalmente, se recomendó el estudio según conclusiones a las que se arriben.

\section{RESULTADOS}

Resultados resaltantes, obtenidas de algunas de las interrogantes de la encuesta; cuya importancia radica en el grado de influencia en los aspectos externos e internos evaluados para esta investigación.
Figura 01: Intención de laborar fuera de la Región

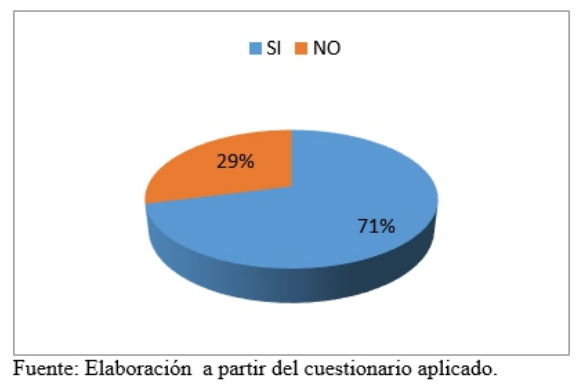

La intención por parte de los estudiantes de laborar fuera de la región es del $71 \%$ mientras que un $29 \%$ prefiere permanecer en el departamento.

Por lo que podemos concluír que la mayoría de los estudiantes prefiere realizar su desarrollo profesional fuera de la región debido a temas de calidad académica y competencia.

Figura 02: Conocimiento de la oficina de Cooperación Internacional en la UNTRM

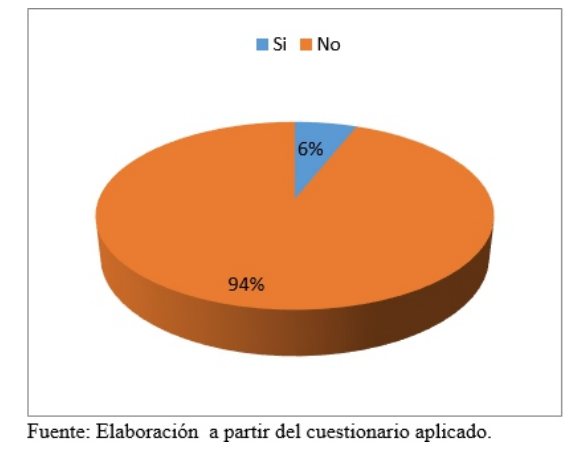

Nos damos cuenta de que el $94 \%$ de los estudiantes no conoce la oficina de cooperación de la Universidad Nacional Toribio Rodríguez de Mendoza, frente a un $6 \%$ que conoce dicha área.

Con lo cual podemos desagregar que la gran mayoría de estudiantes desconoce la presencia de un área administrativa destinada a la cooperación internacional.

Figura 03: Conocimiento relacionado al fortalecimiento profesional a través de la Cooperación

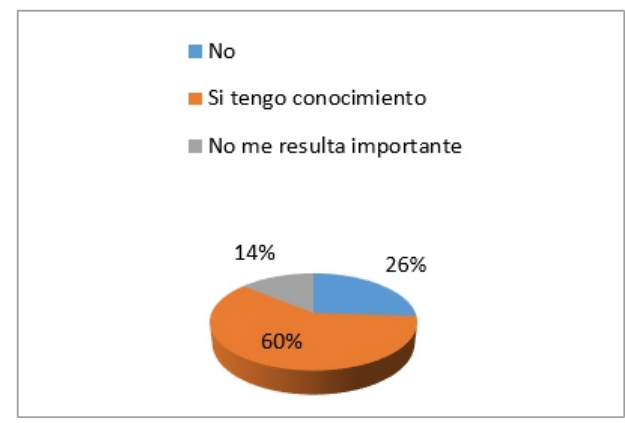

Fuente: Elaboración a partir del cuestionario aplicado. 
Vemos que el $60 \%$ de los estudiantes entrevistados considera y tiene conocimiento de que la cooperación internacional fortalece el desarrollo profesional, mientras que el $26 \%$ considera que no implica cambio alguno en el desempeño de los profesionales.

Por otro lado, un 14\% de los entrevistados considera que no resulta importante la cooperación para la mejora académica.

Figura 04: Universidades o Instituciones con las que la UNTRM debería realizar alianzas.

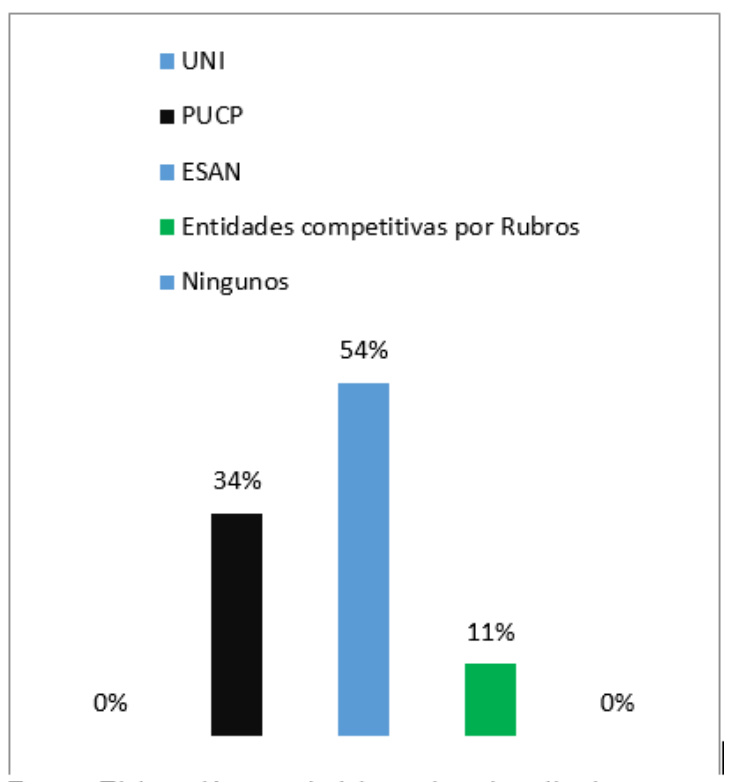

Fuente: Elaboración a partir del cuestionario aplicado.

Los estudiantes de la UNTRM tienen una visión clara de la realidad nacional globalizada y de las principales ventajas que obtendrían realizando cooperación con ciertas instituciones ya reconocidas por su investigación; por tal motivo vemos que el $54 \%$ de los encuestados consideran que la institución con la que se debe realizar cooperación por su calidad es la Universidad ESAN, un 34\% considera que otra institución reconocida es la Pontificia Universidad Católica del Perú; también existe un $11 \%$ de estudiantes que consideran que la mejor elección es establecer cooperación con entidades competitivas dependiendo del rubro en el que nos queramos especializar.

Nos damos cuenta que el conocimiento por parte de los estudiantes con referencia a cuáles instituciones son excelentes para entablar convenios es muy clara, debido al prestigio que han ido adquiriendo al pasar de los años.
Figura 05: Debilidades de la Facultad de Ciencias Económicas y Administrativas

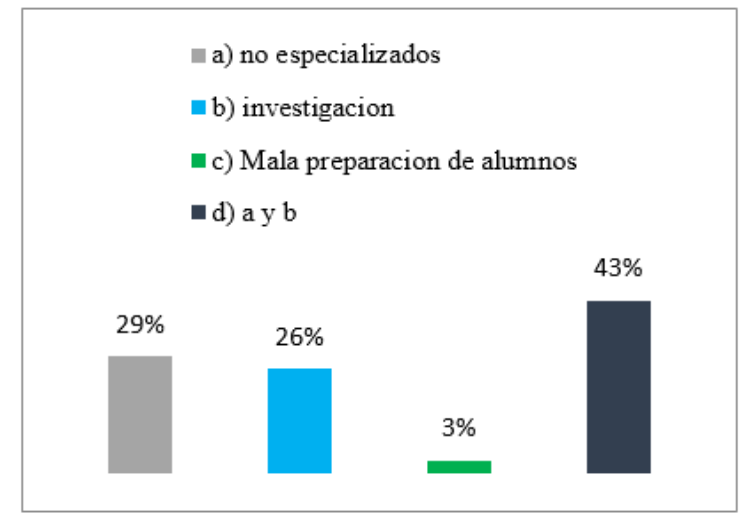

Fuente: Elaboración propia a partir del cuestionario aplicado.

Uno de los aspectos claves de la investigación, ha sido la identificación de las debilidades que presenta la facultad de Ciencias Económicas y Administrativas. Con lo cual se constata que el $43 \%$ de los entrevistados considera que la mayor debilidad de la facultad es que no cuenta con docentes especializados y por ende la mala preparación que reciben los estudiantes, mientras que un $26 \%$ considera que la ausencia de investigación es una de la gran debilidad.

Figura 06: En que se debe especializar un Egresado de Administración de Empresas

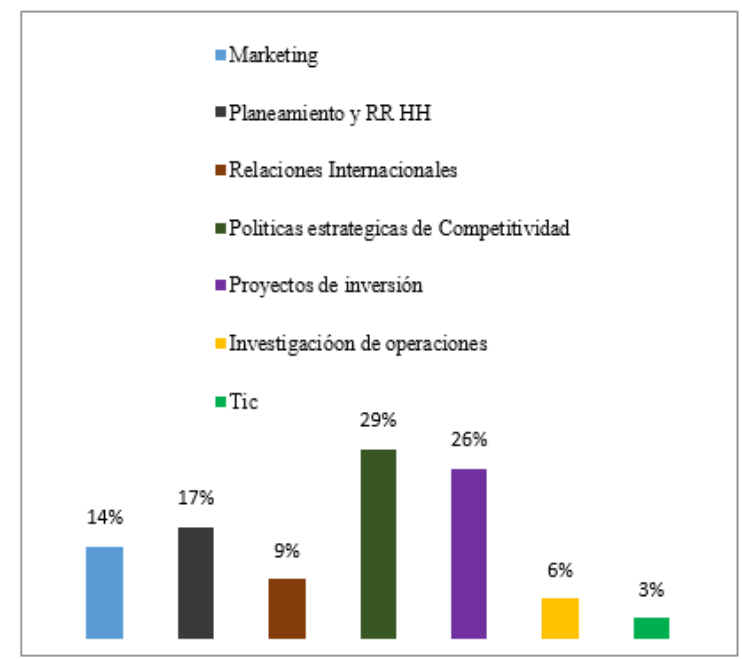

Fuente: Elaboración a partir del cuestionario aplicado.

De los encuestados el $29 \%$ considera que un egresado de la facultad de Ciencias Económicas y Administrativas debe especializarse en políticas estratégicas de cooperación, mientras que una minoría del $3 \%$ considera que deberían especializarse en TIC, esto se debe a la realidad existente actual y aceptable. 
Figura 07: En que se debe especializar un Egresado de Turismo y Hostelería (Administración en Turismo)

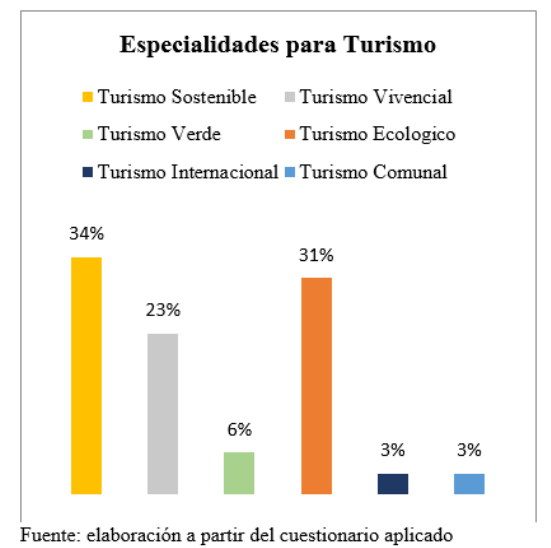

En el caso de los estudiantes de Turismo y Hostelería (Administración en Turismo), consideran que el 34\% de los estudiantes debe especializarse en Turismo Sostenible, mientras que el 3\% debe especializarse en Turismo Comunal.

Considerándose como uno de los mecanismos necesarios que se podrían lograr gracias a la cooperación.

Figura 08: Que convenio es favorable según ámbito

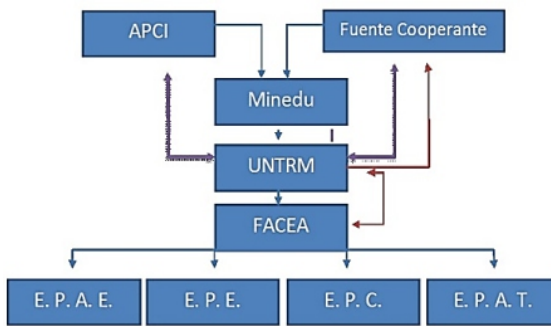

Fuente: elaboracion a partır deI cuestionario apılcado

Los estudiantes, son conscientes de que la mayor parte de oportunidades de desarrollo profesional están presentes en los convenios realizados con empresas de acuerdo a la carrera ya que posibilita la adquisición de experiencia y conocimiento del campo real de acción en el que estarán inmiscuidos una que egresan de las universidades.

Figura 09: Internacionalización (Propuesta estratégica)
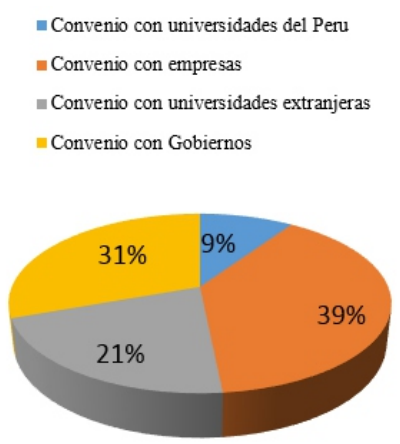

\section{DISCUSIÓN}

En la presente investigación sobre la cooperación se describe el entorno universitario del país, tal es el caso que, el sistema universitario peruano presenta una gran debilidad institucional y de recursos. El objetivo es mejorar la calidad académica y lograr que los estudiantes sean competentes al momento de egresar de las aulas y enfrentarse al mundo profesional.

(CAROLINA, T. \& VIVIANA, G. 2015) en la tesis Cooperación Internacional e Internacionalización en Educación Superior, de la Universidad de San Buenaventura, Cartagena, menciona que las IES utilizan la cooperación internacional para la internacionalización y la mejora de la calidad, además aplica que no toda internacionalización requiere ser parte de la Cooperación Internacional, pero que, toda Cooperación Internacional conlleva a la Internacionalización.

En la FACEA más del $40 \%$ reconoce que la cooperación trae progreso, razón por la que el proceso de globalización genera nuevos mapas productivos, con sus inevitables balances de pérdidas y ganancias (Boisier S., 2005), en su tesis de la Globalización un cambio social en la vida del hombre, explica sobre las alteraciones forzadas del orden social que éstos llevan asociados. Es necesario comprender los cambios continuos a los que está sometido el mundo y sus consecuencias. También es requisito tal como sustentamos se debe fomentar la investigación científica en cooperación, pero por otra parte sostiene (Sebastián J., 2004), en la tesis Impacto de la Cooperación en la calidad de instituciones de educación superior, describe que la globalización ha fortalecido la cooperación en la formación académica de instituciones de educación superior basado en experiencias exitosas producto de estudios causales de las alianzas. También explica cómo se deberían fomentar los proyectos de calidad que incorporen la cooperación como aspecto fundamental en el desarrollo de la calidad.

Integrar la cooperación en el proceso de formación profesional es indispensable. La eficacia de la cooperación radica en una formación de calidad, real y que forme parte del día a día de la Facultad de Ciencias Económicas.

No debemos olvidar que la APCI juega un papel muy importante y que está enmarcado en la Agenda 2030 del desarrollo sostenible con fines de mejorar el bienestar social que se requiere; la disponibilidad de profesionales calificados y con formación de calidad y la capacidad de la sociedad para innovar y adaptar las tecnologías y los conocimientos a un contexto 
nacional específico. La universidad es clave para el desarrollo de la investigación científica y tecnológica. Mediante la investigación aplicada, fomenta nuevas perspectivas y enfoques, a la vez que ayuda a diseñar soluciones localmente relevantes para los problemas de desarrollo económico y social.

El objetivo de la cooperación académica universitaria es que se pueda ampliar la visión profesional de los estudiantes; el $40 \%$ de los estudiantes dice que prefiere hacer especializaciones en alguna rama de su carrera en países de la región Latinoamérica, sin duda un 14\% prefiere ir a Estados Unidos de América a especializarse, pero que el recurso limitante es el dinero para todos los participantes en la recolección de datos.

En las prácticas pre profesionales el 49\% dice que prefiere ir al sector público debido a que es más flexible para iniciarse en el mercado laboral, algunos de ellos señalan que no se encuentran preparados para cumplir horarios complejos y otros explican que no cuentan con la capacidad suficiente en ventajas competitivas para ir al campo privado; entonces (Mera, 2004) explica la necesidad de inserción de cooperación en la universidad y es lógico darle la razón.

Una crítica a los modelos de cooperación en el pasado, ha sido la comprobación de la escasa eficacia de la transferencia de tecnología realizada con ausencia de referencias a las características de los países menos desarrollados, produciendo en muchos casos una dependencia tecnológica. Sin duda, la eficacia de la transferencia tecnológica dependerá del nivel de desarrollo, de las capacidades locales de aplicación, de difusión y de adaptación de tecnologías. La Universidad debe proporcionar, la formación adecuada para lograr la adaptación de la tecnología transferible a las condiciones locales en los programas de desarrollo.

\section{CONCLUSIONES}

En la investigación se acepta que la cooperación impulsa a la mejora de la calidad académica de la universidad.

Es necesario el desarrollo de estrategias de cooperación para alcanzar efectividad en la calidad académica de los estudiantes de la facultad. Así mismo es necesario orientar a la investigación y publicación de la misma, así mismo disponer de un banco de oportunidades gestionado bajo cooperación para beneficios de estudiantes y docente.

La Cooperación es importante para fortalecer el desarrollo de las instituciones, los costos para alcanzar los objetivos pueden ser exuberante sin embargo alianzas y convenios ayudan a reducirlos y alcanzar efectividad a la hora de cumplir metas. Los avances que ha alcanzado la Universidad en diversos rubros de la investigación y los convenios firmados, en un diagnostico sencillo, sirve como base de que la institución está en un plan de internacionalización; trabajo que también se debe realizar para los estudiantes de la FACEA y docentes.

\section{REFERENCIAS BIBLIOGRÁFICAS}

BOISIER, S. (2005). ¿Hay espacio para el desarrollo local en la globalización? Revista de la Cepal, 86(3), pp. 47-62

CAROLINA, T. \& VIVIANA, G. (2015). Cooperación Internacional e Internacionalización en Educación Superior (Tesis de maestría). Universidad de San Buenaventura, Cartagena, Colombia.

MERA, R. (2004). La cooperación universidad empresa - estado en el Perú (Tesis de pregrado). Universidad Nacional Mayor de San Marcos, Lima 